\section{Questioning Decision Analysis for Treatment of Clinically Localized Prostate Cancer}

To the Editor:-Kattan et al. use Monte Carlo simulations of a decision model in their analysis of treatments for clinically localized prostate cancer. ${ }^{1}$ Their application of this approach left me with some questions. The ranges of input probability and quality weight ("utility") parameters reported in Table 3 presumably represent a summary of the distribution of mean values. The result of a Monte Carlo analysis of a decision model using these values is a distribution of expected value for the process. As such, the $95 \%$ confidence interval for this distribution of expected values is range bounded by the 2.5th and 97.5th percentiles of the distribution. The number of Monte Carlo runs used to estimate these values does not affect, as the authors stated, the "statistical significance" of the distribution of these values; rather, it only increases the accuracy with which the confidence intervals are known. In Figure 3, the authors appear to have summarized the confidence interval for the median of the distribution of expected values, something akin to the median of the mean. For me, this has no meaning. To me, the ranges in Figure 3 indicated by the whiskers more likely represent the distributions of the expected values. Thus, for patients with low or moderate cancer grades, the large amount of overlap suggests decision appears to be a toss-up; clinically this makes sense to me. Also, I wonder if the more appropriate measure is the distribution of the difference in expected value between surgery and watchful waiting.-DANIEL $\mathbf{J}$. Cher, MD, General Internal Medicine, Stanford University School of Medicine, Palo Alto, CA.

\section{REFERENCE}

1. Kattan MW, Cowen ME, Miles BJ. A decision analysis for treatment of clinically localized prostate cancer. J Gen Intern Med. 1997; 12:299-305.
In reply:-We appreciate the thoroughness with which Dr. Cher read our paper. He appears to raise three important issues. First, regarding Table 3, the 95\% confidence intervals do not bound the Monte Carlo input parameters. Rather, they help to define the shape of the distribution used for sampling by indicating where much of the data lie.

Dr. Cher's second major point concerns the boxplots of Figure 3, which may be causing confusion. These boxplots have medians, surrounded by a notched area denoting the $95 \%$ confidence interval for the median, which itself is surrounded by the interquartile range (the box), and finally the data range (marked by whiskers). Our concern about statistical significance was that a greater number of simulation runs would result in narrower confidence intervals around each median, increasing the likelihood of detecting differences due to nonoverlapping confidence intervals. We were not interested in detecting small differences between treatments (p. 301). Medians rather than means were illustrated due to the nonnormally distributed data in many of the boxplots in Figure 3.

His third major point concerns illustrating treatment differences rather than individual treatment effectiveness separately. Analysis of the differences obtained in each simulation run would indeed be more statistically powerful because the treatment covariance would be maintained. Unfortunately, our Markov modeling software does not automatically provide that level of detail for the results of second-order Monte Carlo simulations. However, we were able to do one of the scenarios manually, also with 67 model runs. We selected the cohort with the least difference in treatment benefit: the cohort of 75-year-old men with well-differentiated cancer. The boxplot of the treatment benefit for radical prostatectomy appears in Figure 1, presented here. This result agrees with that in the original manuscript: radical prostatectomy provided 0.3 GALYs of benefit. If the treatment decision is a toss-up, our boxplots failed to illustrate it. In any case, a decision analysis such as ours provides very little guidance in the management of the individual prostate cancer patient.-Michael W. Kattan, PhD, Mark E. Cowen, MD, ANd Brian J. Miles, MD, Scott Department of Urology, Baylor College of Medicine, Houston, TX.

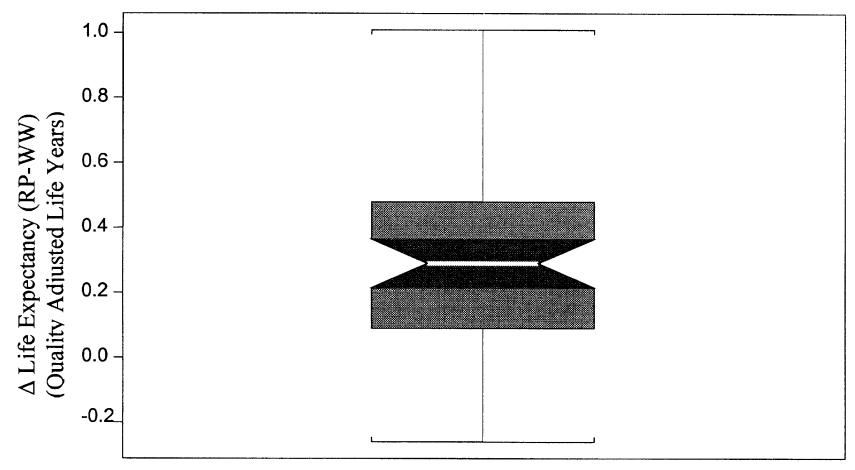

FIGURE 1. Boxplot of the treatment benefit of radical prostatectomy for 75-year-old men with well-differentiated cancer.
JGIM welcomes your letters and comments. Letters may contain brief commentaries on articles published in the Journal, illustrative case reports, general suggestions for improving the Journal, or other information of interest to readers. Letters to the Editor should not exceed 450 words in length and should be sent in triplicate (typed, double-spaced). We cannot guarantee publication and we may edit to improve readability.

You may send your letters to us by mail, fax, or e-mail: JGIM, Veterans Affairs Medical Center (JGIM-111), University and Woodland Avenues, Philadelphia, PA 19104; fax: 215-8234450; e-mail:jgim@mail.med.upenn.edu.. 